\title{
Increased NIH 3T3 fibroblast functions on cell culture dishes which mimic the nanometer fibers of natural tissues
}

This article was published in the following Dove Press journal:

International Journal of Nanomedicine

24 August 2015

Number of times this article has been viewed

\author{
Garima Bhardwaj' \\ Thomas J Webster ${ }^{1,2}$ \\ 'Department of Chemical Engineering, \\ Northeastern University, Boston, \\ MA, USA; ${ }^{2}$ Center of Excellence \\ for Advanced Materials Research, \\ King Abdulaziz University, Jeddah, \\ Saudi Arabia
}

\begin{abstract}
Traditional flat tissue cell culture dishes have consisted of polystyrene treated with plasma gases for growing, subculturing, and studying cell behavior in vitro. However, increasingly it has been observed that mimicking natural tissue properties (such as chemistry, three-dimensional structure, mechanical properties, etc) in vitro can lead to a better correlation of in vitro to in vivo cellular functions. The following studies compared traditional NIH 3T3 fibroblasts' functions on XanoMatrix scaffolds to standard tissue culture polystyrene. Results found significantly greater fibroblast adhesion and proliferation on XanoMatrix cell culture dishes which mimic the nanoscale geometry of natural tissue fibers with true, tortuous fiber beds creating a robust, consistent, and versatile growth platform. In this manner, this study supports that cell culture dishes which mimic features of natural tissues should be continually studied for a wide range of applications in which mimicking natural cellular functions are important.
\end{abstract}

Keywords: nanotechnology, cell culture, fibroblasts

\section{Introduction}

Drug discovery programs require accurate in vitro systems for drug screening and testing. Traditional cell culture makes use of two-dimensional (2D) surfaces for ex vivo cell growth. In such environments, cells are forced to adopt unnatural characteristics, including aberrant flattened morphologies. Cell-based in vitro assays are a key component of drug discovery research. Cultured mammalian cells are important tools for providing predictions of drug activity, metabolism, and toxicity in vivo. Therefore, there is a strong demand for new cell culture platforms which allow cells to grow and respond to their environment in a more realistic manner. However, traditional cell culture environments are far removed from real-life tissues. In vivo, cells are supported by a complex three-dimensional (3D) extracellular matrix, which facilitates cell-cell communication via direct contact and through the secretion of a plethora of cytokines and trophic factors. In contrast, cells grown in culture are generally confined in 2D monolayers without many of the physical and chemical cues which underlie their identity and function in vivo. In vitro, cells can behave very differently depending on the growth substrate employed. Conventional tissue culture is carried out on 2D surfaces without scope for cells to adopt natural morphologies or to communicate efficiently with their neighbors. ${ }^{1}$ It has been shown using alternative cell culture applications that the growth and function of cells as multicellular 3D structures is significantly different to their growth as conventional 2D monolayer cultures. ${ }^{2}$ This $2 \mathrm{D}$ confinement is far removed from the aforementioned 3D complexities of living tissue. ${ }^{3}$ Although there are a variety of technologies
Correspondence: Thomas J Webster Department of Chemical Engineering, Northeastern University, 360 Huntington Ave, Boston, MA 02115 USA

Email th.webster@neu.edu 
available that enable 3D cell growth, most of these have been developed for transplantation purposes and tissue engineering in vivo. Comparatively less attention has been devoted to the development of 3D culture systems for exclusive use in the laboratory as an approach to improving the accuracy of in vitro analyses. A range of materials have been considered for $3 \mathrm{D}$ cell growth supports in vitro. These include naturally occurring materials as well as products fabricated from naturally derived and synthetic polymers. Natural substrates such as alginate, which is a seaweed-derived material, have been used to support cell growth in a number of ways including cell encapsulation. ${ }^{4}$ Granted that this technology does enable a degree of 3D cell growth, growing cells as individual spherical masses is not necessarily suitable for all requirements as the distribution of cells throughout the material is not entirely even, and there are issues about mass transfer given the thickness of the scaffold under static growth conditions. Furthermore, it is not clear whether cells will respond differently to the alginate substrate compared with conventional 2D plastic ware, which is familiar and has been used for many years. An alternative and one of the early most successful approaches has been to culture cells on biodegradable polymers such as poly(glycolic acid), poly(lactic acid), and their copolymers poly(lactic-coglycolic acid). ${ }^{5}$ Hydrogels are a common form of a material that has successfully been used to support ex vivo $3 \mathrm{D}$ cell growth for a variety of systems, such as bone, cartilage, and nervous system tissues. ${ }^{6}$

Nanotechnology refers to materials whose diameters are $<1,000$ nanometers. $^{7}$ Many remarkable characteristics have been observed when comparing nanomaterials to conventional (or micron-structured) materials such as very large surface-area-to-volume ratios, flexibility in surface functionalities due to this greater surface area, and superior mechanical performance including stiffness and tensile strength. ${ }^{8,9}$ Of course, one of the most appealing properties of nanomaterials compared to conventional materials is in regenerative medicine since nanomaterials mimic the natural dimensions of tissue constituents. Such biologically-inspired properties of nanomaterials have led to their use in improving bone, cartilage, nervous system, bladder, vascular, skin, and numerous other tissue applications. Moreover, the application of nanomaterials (such as nanofibers) has included membranes for gas filtration, water purification, and sound absorption mats. ${ }^{10}$ Nanofiber production has gained considerable momentum worldwide due to high surface area and intriguing properties. Consequently, much research has also been conducted to optimize nanofiber processing techniques such as drawing, template synthesis, phase separation, selfassembly, electrospinning, etc.

Unlike conventional electrospinning methods of creating nanofibers with expensive machines using an electrical charge to draw individual fibers to a grounded surface, XanoShear ${ }^{\mathrm{TM}}$ (Xanofi, Raleigh, NC, USA) is a technology that is elegant in its simplicity and efficient in its design. Originally developed with a device with two concentric cylinders, this technology is capable of creating nanofibers through a combination of shear force and polymer phase separation without the use of nozzles or spinnerets.

The fibers are synthesized by introducing a polymer solution in the bulk of a viscous medium under shear. The medium is chosen so that it is miscible with the polymer solvent, but also precipitates the polymer. The breakthrough in the ability to draw very thin fibers comes from the ultralow interfacial tension between the droplets and the medium, which allows for a high degree of stretching and generation of materials with high surface area. As the solution droplets are highly stretched in parallel by shear, the solvent diffuses out of the droplets leaving behind polymer fibers in the diameter range of $50 \mathrm{~nm}-2 \mu \mathrm{m}$. The shear process can be used in the fabrication of nanofibers from many classes of materials, including hydrophilic, chemically or biologically active polymers. The resulting polymer nanofibers can also incorporate functional particles by simply adding them to the polymer solution.

Due to the above and the relatively unstudied application of nanofibers as improved cell culture substrates, the objective of the present study was to determine the functions of a well-established cell line, NIH 3T3 fibroblasts, on a biologically-inspired nanofibrous XanoMatrix compared to traditional tissue culture polystyrene dishes. Improving cell functions on tissue cell culture dishes to match that on natural materials, could lead to improved identification of medical devices and drug delivery materials as well as an understanding of disease prevention, diagnosis, and treatment. XanoMatrix is currently made with biocompatible materials polyethylene terephthalate (PET) and cellulose acetate (CA). PET, also known as Dacron, has been widely used as prosthetic vascular grafts and has shown excellent mechanical strength and good biocompatibility. ${ }^{11} \mathrm{CA}$ is an industrially important cellulose ester with good mechanical and wetting properties. CA nanofibers have also increasingly been used in tissue engineering. ${ }^{12}$ XanoMatrix scaffolds combine the advantages of nano-sized polymeric fibers with true, tortuous fiber beds and supports to create a robust, consistent, and versatile growth platform that 
properly mimics native tissue. XanoMatrix scaffolds can be easily placed in traditional 6-well, 48-well, and 96-well formats as well as $10 \times 11^{\prime \prime}$ sheets for custom shapes and sizes.

\section{Materials}

The PET and CA nano-fiber scaffolds (XanoMatrix) were fabricated by XanoShear ${ }^{\mathrm{TM}}$ technology as described above (Figure 1). Sterile 6-, 48-, and 96-well cell culture dishes were obtained from Xanofi for studies below. Sterile traditional plasma treated polystyrene cell culture dishes were obtained from Corning and Falcon (Corning Incorporated, Corning, NY, USA) for comparative analysis. NIH 3 T3 fibroblasts $\left(\right.$ ATCC $^{\circledR}$ 30-2003 ${ }^{\text {TM }}$ [American Type Culture Collection, Manassas, VA, USA]) were used to perform cellular adhesion and proliferation studies as described below.

\section{Methods}

\section{Surface characterization}

Atomic force microscopy

The surface morphology of the samples was analyzed by using a Parks Scientific NX-10 atomic force microscope in non-contact mode and the root mean square surface roughness was determined.

\section{Contact angle analysis}

Contact angle analysis was used to test the hydrophilicity/ hydrophobicity using the Pioneer contact angle 300 goniometer.

\section{Scanning electron microscopy}

The surface features of the samples were visualized using a Hitachi $4800-S$ scanning electron microscope at a voltage of $5.0 \mathrm{kV}$ and a magnification of 250 .

\section{Cell culture}

NIH 3 T3 fibroblasts were cultured using Eagle's Minimum Essential Medium (ATCC ${ }^{\circledR}$ 30-2003 ${ }^{\mathrm{TM}}$ ) supplemented with $10 \%$ fetal bovine serum (ATCC ${ }^{\circledR}$ SCRR-30-2020 ${ }^{\mathrm{TM}}$ ) and a $1 \%$ penicillin-streptomycin solution (ATCC ${ }^{\circledR} 30-2300^{\mathrm{TM}}$ ). Cell adhesion and proliferation was determined using MTS assays after 1, 7, 14, and 21 days. The cells were seeded at 15,000 cells $/ \mathrm{cm}^{2}$ for the adhesion assays and at $3,500 \mathrm{cells} / \mathrm{cm}^{2}$ for the proliferation assays. For the proliferation assay, the media was changed every other day. The MTS (CellTiter $96^{\circledR} \mathrm{AQ}_{\text {ueous }}$ One Solution Cell Proliferation Assay, G3581; Promega Corporation, Fitchburg, WI, USA) reagent (1:5 ratio with cell culture media) was added to each well and incubated for 3 hours on the day of the measurement. At the end of incubation, a color change from pink to dark brown was observed, and absorbance from each well was measured by a SpectraMax M3 (MT05412) at $490 \mathrm{~nm}$.

\section{Confocal microscopy}

To further verify the data collected by adhesion and proliferation assays, confocal microscopy was used to visualize the surface after growing the cells, fixing the cells using glutaraldehyde followed by successive dehydration using $50 \%, 70 \%, 90 \%$, and $100 \%$ ethanol and then staining with staining with $20 \mathrm{~nm}$ Syto 9 dye.

\section{Statistical analysis}

All experiments were completed in triplicate and repeated at least three different times.

\section{Results and discussion}

The surface of the XanoMatrix was found to be more rough than the Corning and Falcon petri dishes (Figure 2). The XanoMatrix surface was more hydrophobic in nature as compared to the Corning and Falcon petri dishes (Figure 3).

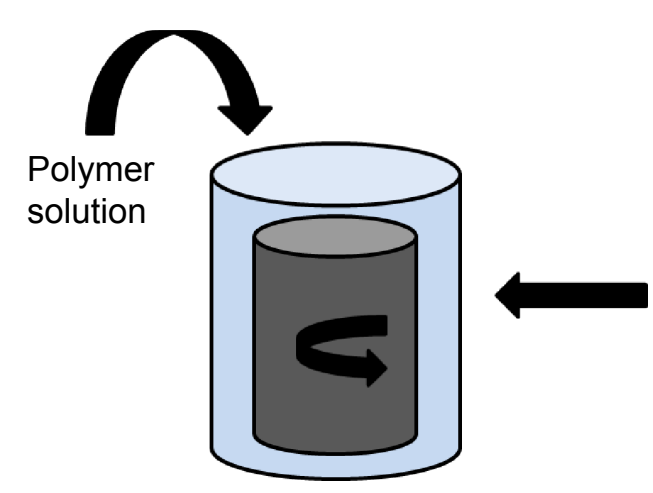

Viscous medium under

shear stress. Miscible with

polymer solvent but also

precipitates polymer

Figure I Basic schematic of the XanoShear ${ }^{\mathrm{TM}}$ (Xanofi, Raleigh, NC, USA) process. 

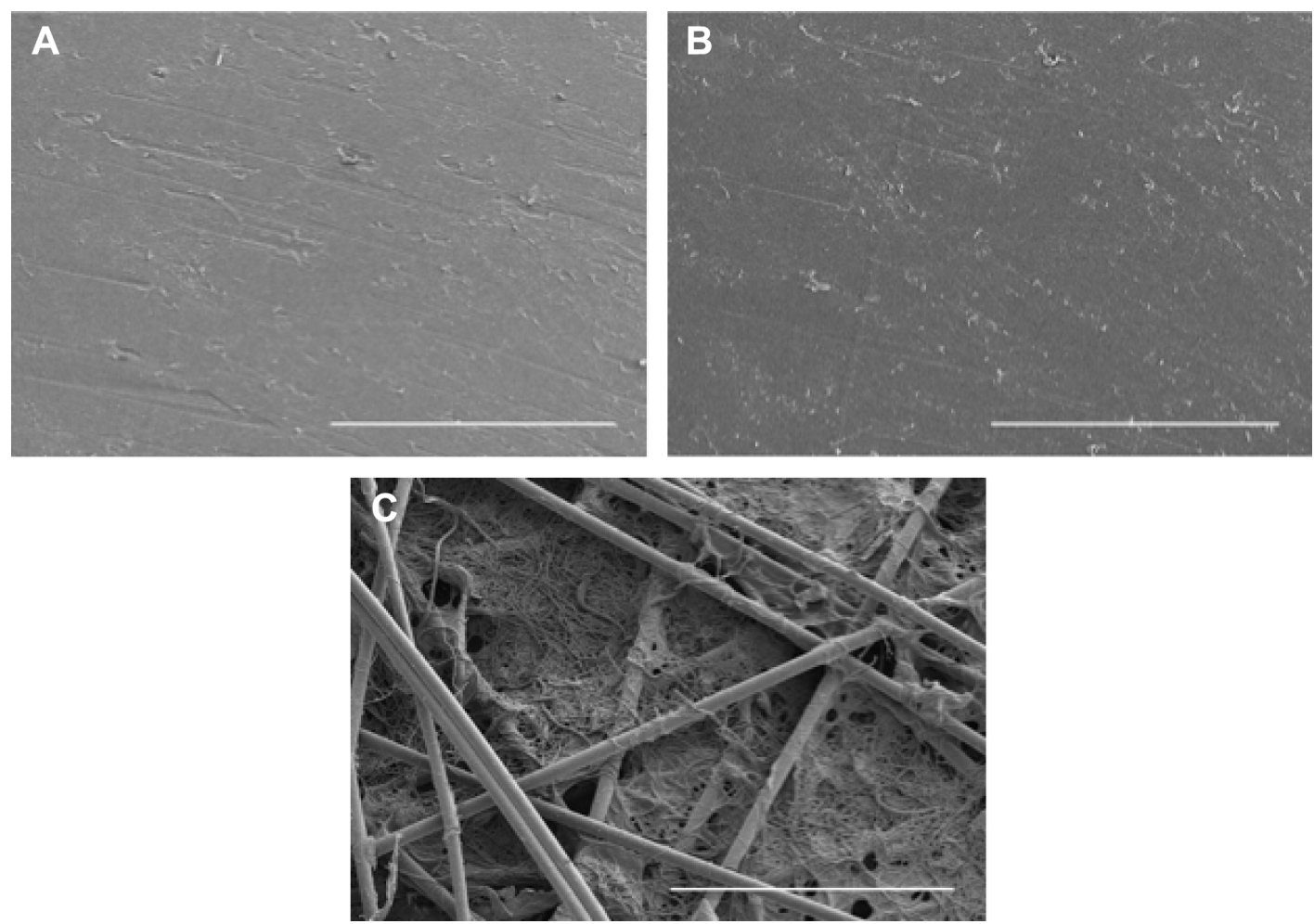

Figure 2 Scanning electron microscopy images.

Notes: Scanning electron microscopy images of the surfaces of (A) Corning; (B) Falcon; and (C) XanoMatrix surfaces of the cell culture petri dishes. Scale bar $=500$ microns.

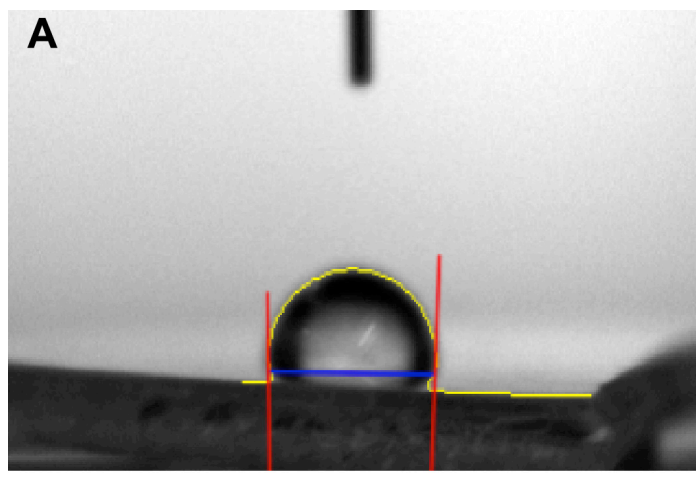

\section{B}

C
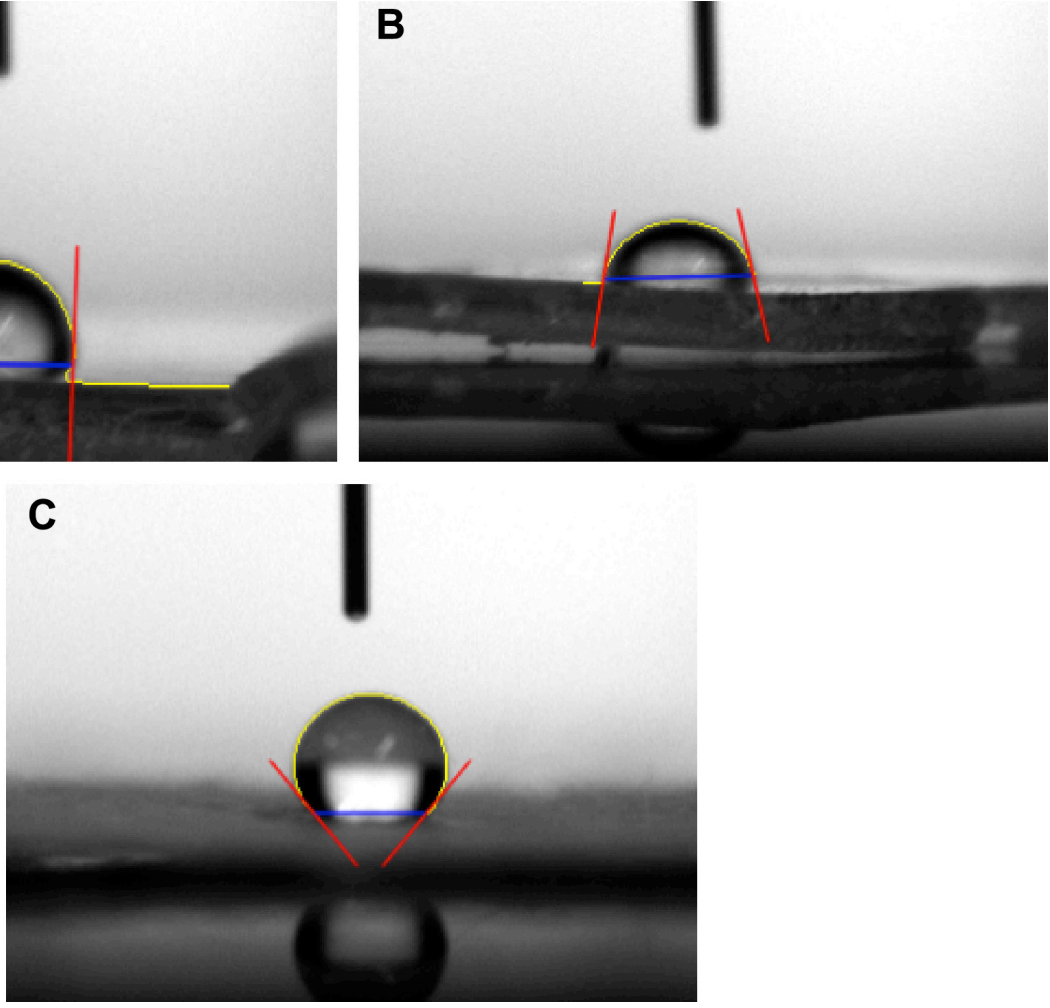

Figure 3 Contact angle images.

Notes: Contact angle images on the surfaces of: (A) Corning (91.3157 degrees); (B) Falcon (80.1424 degrees); and (C) XanoMatrix (129.3827 degrees). 
The surface of the XanoMatrix showed the presence of nanofibers and was $3 \mathrm{D}$ in nature as compared to the Corning and Falcon petri dishes (Figure 4). Thus, it was more replicative of an in vivo environment for cell growth and proliferation.

In vitro, cells can behave very differently depending on the growth substrate employed. Conventional tissue culture is carried out on 2D surfaces without the ability of cells to adopt natural morphologies or to communicate efficiently with their neighbors. This $2 \mathrm{D}$ confinement is far removed from the 3D complexities of living tissue. Engineering the cell culture microenvironment to create growth conditions that more accurately mimic the in vivo behavior of cells is an essential step for improving predictive accuracy during pharmaceutical development/clinical trials.

As expected, here, the XanoMatrix cell culture well plates increased fibroblast adhesion and proliferation as compared to the Corning and Falcon cell culture petri dishes since they offered a more biologically-inspired 3D nanofiber structure or a structure that mimics the extracellular matrix in the body (Figures 5 and 6). The XanoMatrix surface also had increased roughness at the nanoscale and hydrophobicity as compared to the other control surfaces used in this study. The interaction between fibroblasts and nanofibrous polymers plays an important role in biotechnology and biomedical applications.

Around the world, the increasing use of polymeric materials in these applications ranges from substrates for cell and tissue growth to vascular and other numerous prostheses. Materials for tissue and cell culture are not inert polymers without biological relevance, as it is known that the cellular response is driven by polymer surface characteristics such as topography, hydrophobic/hydrophilic properties, and chemistry. ${ }^{13}$ Improvements in biocompatibility due to surface modification is mainly based on incorporating new functional chemical groups, changes in polarization and surface free energy, as well as changes in topography. ${ }^{14}$ The 3D growth of cells (as observed here) opens up the possibility of true 3D migration, invasion, and nutrient exchange as seen in native tissues. Synthetic polymeric nanofibers are increasingly being used to imitate these structures for
A

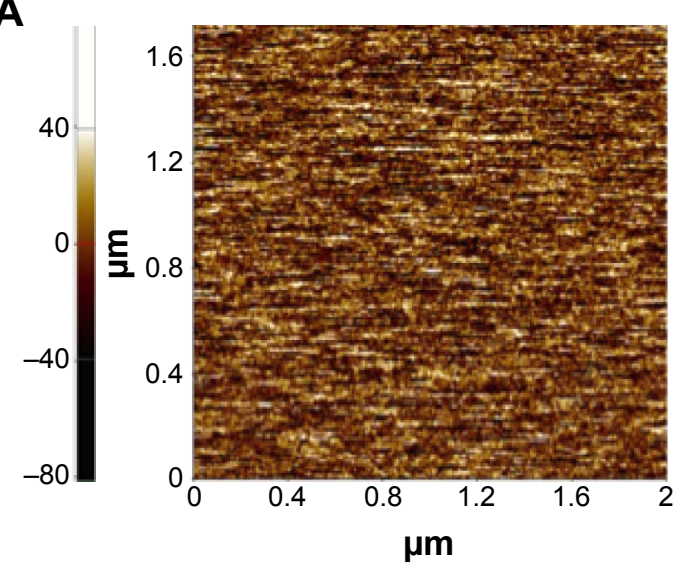

B

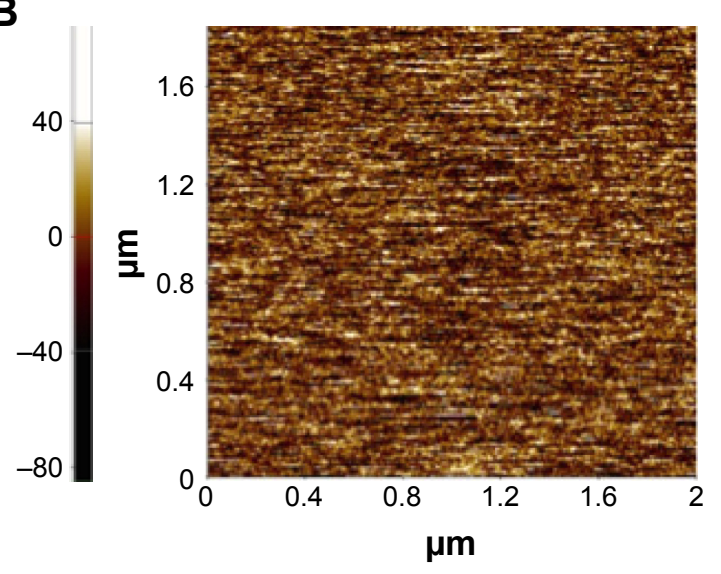

C

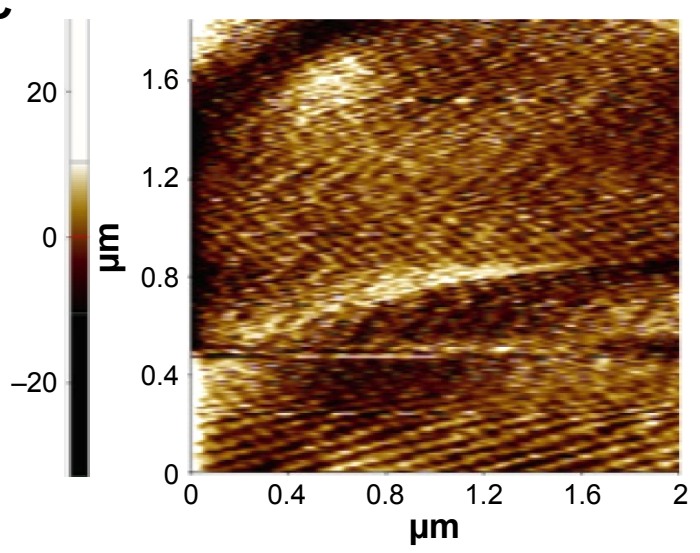

Figure 4 Atomic force microscopy images.

Notes: Atomic force microscopy images of the surfaces of (A) Corning; (B) Falcon; and (C) XanoMatrix. Surface roughness was $27.84 \mathrm{~nm}, 30.027 \mathrm{~nm}$, and $62.182 \mathrm{~nm}$ for (A) Corning; (B) Falcon; and (C) XanoMatrix, respectively. 


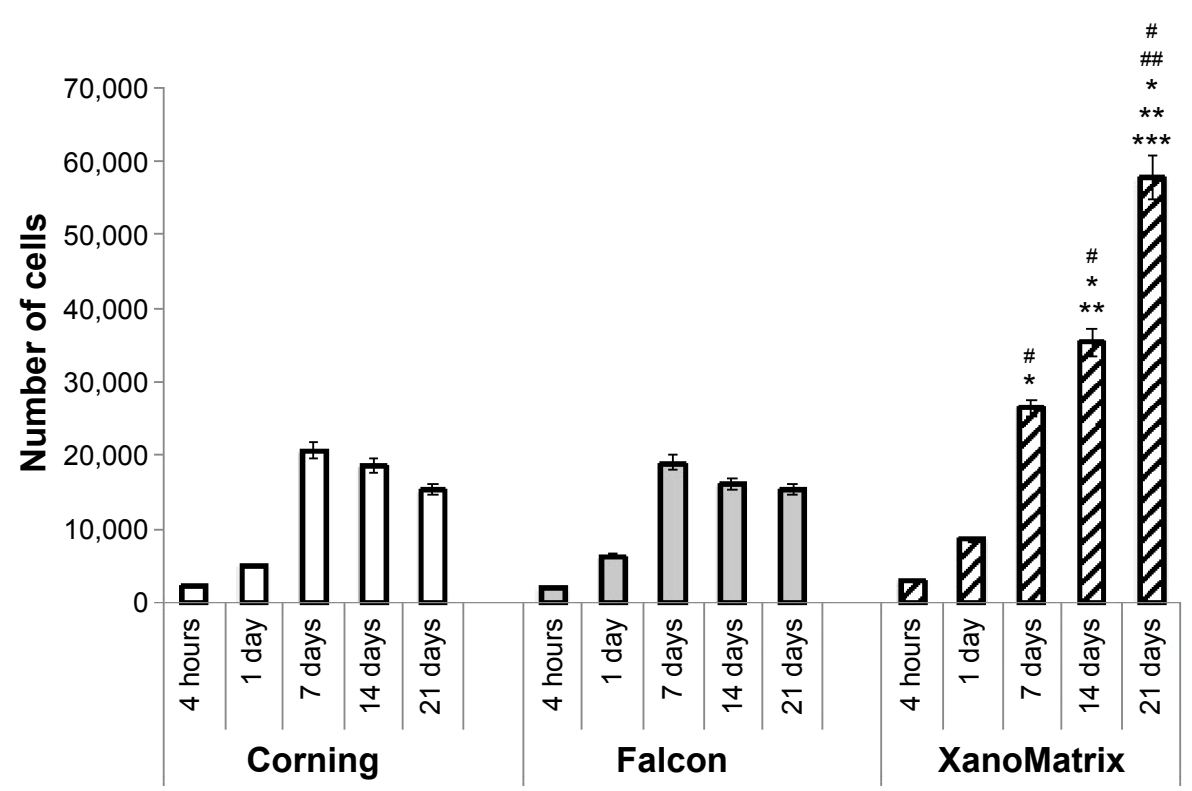

Figure $5 \mathrm{NIH} 3 \mathrm{~T} 3$ fibroblast adhesion and proliferation on Corning, Falcon, and XanoMatrix cell culture petri dishes after 4 hours as well as I, 7, I4, and $2 \mathrm{I}$ days of culture.

Notes: Data are expressed as the mean \pm standard error of the mean; $N=3 ; * P<0.01$ as compared to Corning and Falcon petri dishes on the 7 th day of culture. $* * P<0.01$ as compared to the Corning and Falcon petri dishes on the I4th day of culture. ${ }^{*} * *<0.0 \mathrm{I}$ as compared to Corning and Falcon petri dishes on the 2 I st day of culture. $\# P<0.0 \mathrm{I}$ as compared to XanoMatrix cell culture petri dishes on Ist day of cell culture. $\# P<0.01$ as compared to XanoMatrix cell culture petri dishes on 7 th day of cell culture.

research related to tissue engineering, cancer, stem cells, high-throughput cell culture, and regenerative medicine applications. Nanofibers have an advantage over traditional scaffolds and gels because of their lack of animal-derived products, batch-to-batch consistency, ability to be shaped into various forms, and structural and size similarity to native tissue. Traditional electrospun nanofibers produce a flat, webbed matrix that on the surface mimics native extracellular matrices, but often does not allow deep penetration of cells into the structure. Porous or foamed plastic allows z-axis cellular growth, but still does not create a fibrous surface for the cells to grow more naturally and remodel. XanoMatrix scaffolds combine the advantages of nano-sized polymeric fibers with true, tortuous fiber beds and supports to create a robust, consistent, and versatile growth platform that properly mimics native tissue.

\section{Conclusion}

This study shows that replacing the use of traditional tissue culture treated polystyrene petri dishes with more biologically-inspired XanoMatrix scaffolds provided a better environment for the adhesion and growth of fibroblasts. In this manner, this study supports that cell culture dishes which mimic features of natural tissues should be continually studied for a wide range of applications in which mimicking natural cellular functions are important.
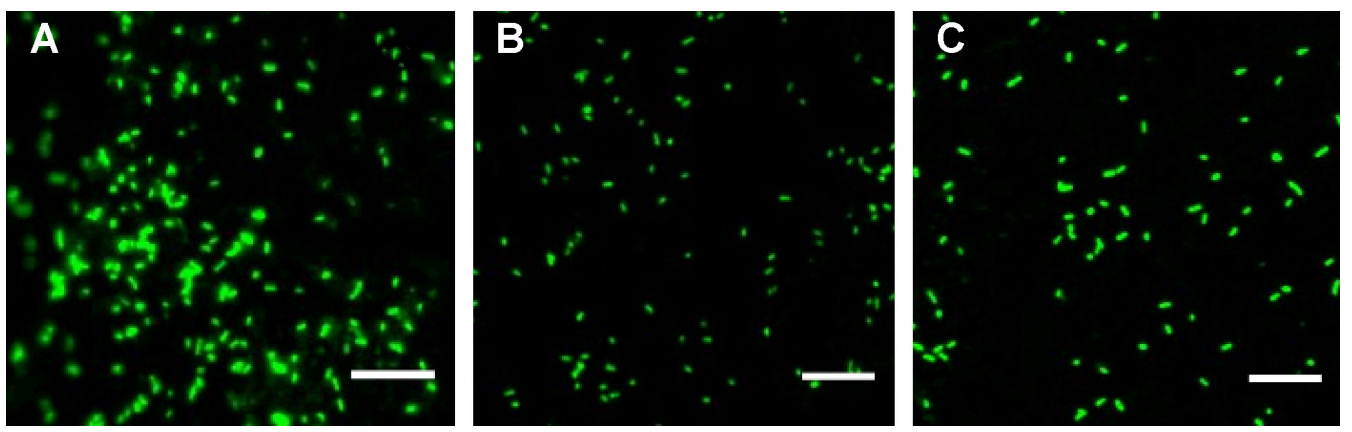

Figure 6 Confocal microscopy images of NIH 3T3 fibroblast adhesion.

Notes: Confocal microscopy images of NIH $3 \mathrm{~T} 3$ fibroblast adhesion on day 7 on (A) XanoMatrix; (B) Falcon; and (C) Corning surfaces. Scale bar =60 microns. Magnification I0X. 


\section{Acknowledgment}

The authors would like to thank Xanofi for providing the materials for the study and for funding.

\section{Disclosure}

The authors have no conflicts of interest to disclose.

\section{References}

1. Bhadriraju K, Chen CS. Engineering cellular microenvironments to improve cell-based drug testing. Drug Discov Today. 2002;7(11): 612-620.

2. Schmeichel KL, Bissell MJ. Modeling tissue-specific signaling and organ function in three dimensions. J Cell Sci. 2003;116(Pt 12):2377-2388

3. Maltman DJ, Przyborski SA. Developments in three-dimensional cell culture technology aimed at improving the accuracy of in vitro analyses. Biochem Soc Trans. 2010;38(4):1072-1075.

4. de Vos P, Faas MM, Strand B, Calafiore R. Alginate-based microcapsules for immunoisolation of pancreatic islets. Biomaterials. 2006;27(32): $5603-5617$.

5. Mikos AG, Sarakinos G, Leite SM, Vacanti JP, Langer R. Laminated 3-dimensional biodegradable foams for use in tissue engineering. Biomaterials. 1993;14(5):323-330.

6. Blackshaw SE, Arkison S, Cameron C, Davies JA. Promotion of regeneration and axon growth following injury in an invertebrate nervous system by the use of three-dimensional collagen gels. Proc Biol Sci. 1997; 264(1382):657-661.
7. Maheshwari SU, Kumar SV, Nagiah N, Uma TS. Electrospinning of polyvinylalcohol-polycaprolactone composite scaffolds for tissue engineering applications. Polymer Bulletin. 2013;70(11):2995-3010.

8. Ma Z, Kotaki M, Yong T, He W, Ramakrishna S. Surface engineering of electrospun polyethylene terephthalate (PET) nanofibers towards development of a new material for blood vessel engineering. Biomaterials. 2005;26(15):2527-2536.

9. Savoji H, Hadjizadeh A, Maire M, Ajji A, Wertheimer MR, Lerouge S. Electrospun Nanofiber Scaffolds and Plasma Polymerization: A Promising Combination Towards Complete, Stable Endothelial Lining for Vascular Grafts. Macromol Biosci. 2014;14(8):1084-1095.

10. Huang ZM, Zhang Y, Kotaki M, Ramakrishna S. A review on polymer nanofibers by electrospinning and their applications in nanocomposites. Composites Science and Technology. 2003;63(15):2223-2253.

11. Dewez JL, Doren A, Schneider YJ, Rouxhet PG. Competitive adsorption of proteins: Key of the relationship between substratum surface properties and adhesion of epithelial cells. Biomaterials. 1999;20(6):547-559.

12. Arbeitman CR, del Grosso MF, Ibanez I, et al. Irradiation of Polystyrene and Polypropylene to study NIH 3T3 fibroblasts adhesion. Nuclear Instruments and Methods in Physics Research Section B: Beam Interactions with Materials and Atoms. 2010;268(19):3059-3062.

13. Li Y, Ma T, Yang ST, Kniss DA. Thermal compression and characterization of three-dimensional nonwoven PET matrices as tissue engineering scaffolds. Biomaterials. 2001;22(6):609-618.

14. Ali S, Khatri Z, Oh KW, Kim IS, Kim SH. Zein/cellulose acetate hybrid nanofibers: Electrospinning and characterization. Macromolecular Research. 2014;22(9):971-977.
International Journal of Nanomedicine

\section{Publish your work in this journal}

The International Journal of Nanomedicine is an international, peerreviewed journal focusing on the application of nanotechnology in diagnostics, therapeutics, and drug delivery systems throughou the biomedical field. This journal is indexed on PubMed Central,

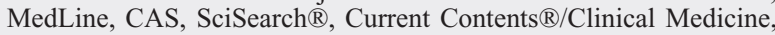

\section{Dovepress}

Journal Citation Reports/Science Edition, EMBase, Scopus and the Elsevier Bibliographic databases. The manuscript management system is completely online and includes a very quick and fair peer-review system, which is all easy to use. Visit http://www.dovepress.com/ testimonials.php to read real quotes from published authors. 